\title{
DIAGNÓSTICO DA MORBIDADE E MORTALIDADE DOS CASOS DE LEPTOSPIROSE NO NORDESTE BRASILEIRO ENTRE 2000 A 2015
}

Yara Soares dos Santos ${ }^{1}$, Elania Barros da Silva², José Francisco de OliveiraJúnior $^{3}$, Paulo José dos Santos ${ }^{4}$, Luana Maria Barros Costa ${ }^{5}$

1. Estudante de Pós-Graduação em Meteorologia da Universidade Federal de Alagoas (UFAL), Maceió/AL - Brasil.

2. Enfermeira, Secretaria de Saúde de Capela, Capela/AL - Brasil.

3. Professor Doutor do Instituto de Ciências Atmosféricas (ICAT) da

Universidade Federal de Alagoas (UFAL), Maceió/AL - Brasil.

jose.junior@icat.ufal.br

4. Mestre em Meteorologia do Instituto de Ciências Atmosféricas (ICAT) da

Universidade Federal de Alagoas (UFAL), Maceió/AL - Brasil.

5. Estudante de Serviço Social da Universidade Federal de Alagoas (UFAL), Maceió/AL - Brasil.

Recebido em: 06/04/2018 - Aprovado em: 10/06/2018 - Publicado em: 20/06/2018 DOI: 10.18677/EnciBio_2018A34

\section{RESUMO}

O objetivo do estudo foi realizar um diagnóstico da morbidade e mortalidade dos casos de Leptospirose no Nordeste Brasileiro (NEB). A série temporal de leptospirose de 2000 a 2015 foi obtida do DATASUS, sendo avaliada via estatística descritiva e exploratória. Os resultados obtidos da estatística descritiva aplicada à série temporal mostraram que os anos $(2000,2004,2009,2010$ e 2011) e os estados de PE (4506 casos, 41,10\%), seguido da BA (2220 casos, $20,25 \%)$, CE (1248 casos, $11,38 \%)$ e $\mathrm{AL}(1209$ casos, $11,03 \%)$ tiveram um aumento significativo de morbidade de leptospirose. No caso da mortalidade, ocorreram nos anos 2000 (114 casos, $11.54 \%$ ), 2004 (120 casos, 7,36\%), 2009 (85 casos, 6,20\%), 2010 (76 casos, 5,54\%) e 2011 (118 casos, 8,61\%), respectivamente. A estatística exploratória identificou que os casos de morbidade e mortalidade de leptospirose no NEB são assimétricos positivos em relação à série temporal, com exceções os anos de 2001, 2008 e 2014. Com base na estatística, foi demonstrado que os estados de $\mathrm{PE}, \mathrm{BA}, \mathrm{CE}, \mathrm{AL}$ e SE se sobressaem em relação aos demais estados, em virtude dos ciclos e eventos extremos pluviométricos que neles ocorreram ao longo da série temporal, juntamente, com processo de urbanização e crescimento populacional.

PALAVRAS-CHAVE: Saúde Pública, Série Temporal, Sistema Meteorológicos, Urbanização.

\section{DIAGNOSIS OF MORBIDITY AND MORTALITY OF LEPTOSPIROSE CASES IN NORTHEAST BRAZIL BETWEEN 2000 TO 2015}

\begin{abstract}
The objective of the study was to perform a diagnosis of the morbidity and mortality of Leptospirosis cases in the Brazilian Northeast (NEB). The time series of leptospirosis from 2000 to 2015 was obtained from DATASUS, being evaluated through descriptive and exploratory statistics. The results obtained from the descriptive statistics applied to the time series showed that the years $(2000,2004$,
\end{abstract}


2009, 2010 and 2011) and the states of PE (4506 cases, $41.10 \%)$ followed by BA (2220 cases, $20.25 \%$ ), CE (1248 cases, 11.38\%) and AL (1209 cases, $11.03 \%$ ) had a significant increase in the morbidity of leptospirosis. In the case of mortality, in 2000 (114 cases, 11.54\%), 2004 (120 cases, 7.36\%), 2009 (85 cases, 6.20\%), 2010 (76 cases, 5.54\%) and 2011 (118 cases, 8.61\%), respectively. The exploratory statistics identified that the cases of morbidity and mortality of leptospirosis in the NEB are asymmetric positive in relation to the time series, except for the years of 2001, 2008 and 2014. Based on the statistics, was demonstrated the states of PE, $\mathrm{BA}, \mathrm{CE}, \mathrm{AL}$ and $\mathrm{SE}$ stand out in relation to the other states, due to the cycles and extreme rainfall events that occurred during the time series, together with urbanization and population growth.

KEYWORDS: Public Health, Meteorological Systems, Time Series, Urbanization.

\section{INTRODUÇÃO}

Atualmente, o crescimento urbano desordenado, vem sendo tema de grandes discussões no âmbito acadêmico e pelos gestores de políticas públicas, quanto ao impacto do clima urbano na saúde (SOUSA et al., 2015; HAGAN et al., 2016). Associado ao processo de urbanização está também o crescimento populacional não acompanhado de planejamento por parte dos gestores públicos, que deu origem as favelas (CLEMENTE; OLIVEIRA-JÚNIOR, 2015). As favelas são áreas insalubres sujeitas a condições de risco, e ao surgimento de doenças e epidemias causadas pela falta de higiene e serviços sanitários (NOBRE et al., 2010; GUIMARÃES et al., 2014). Em virtude dessas condições, tais populações estão mais vulneráveis aos impactos negativos de ordem social e ambiental (GONÇALVES et al., 2016).

No Brasil, as inundações/enchentes é um dos problemas recorrentes em virtude da falta de planejamento urbano (NOBRE et al., 2010; GOULART et al., 2015). As inundações/enchentes são consequências de fortes chuvas nos ambientes urbanos e assolam as populações, com isso, favorece o aumento e a migração de vetores de epidemias e doenças, e assim, traz sérios riscos de saúde (COELHO-ZANOTTI; MASSAD, 2012). Anteriormente, a doença estava relacionada às áreas rurais, porém, com o aumento da urbanização, passou a ser associada com determinantes sociais, tais como, pobreza, submoradia e acesso limitado aos serviços de coleta de lixo, esgotamento sanitário e água potável (DUTRA et al., 2015).

As inundações/enchentes que ocorrem nos períodos chuvosos têm aumentado os casos de leptospirose no Brasil, principalmente, em Regiões Metropolitanas (RM) (COELHO-ZANOTTI; MASSAD, 2012; GUIMARÃES et al., 2014). Para Sousa et al. (2015), o não planejamento de forma adequada e a ocupação desordenada são agravantes do problema das inundações urbanas. Assim, quando as cidades não possuem um sistema de tratamento pluvial e de redes de esgotos adequados, bem como uma coleta de lixo periódica, a leptospirose aumenta substancialmente, uma vez que os vetores da doença encontram nestes ambientes, condições propícias para se proliferarem (PELISSARI et al., 2011).

O agente da leptospirose é uma bactéria helicoidal (espiroqueta) aeróbica obrigatória do gênero Leptospira (CONFALONIERI, 2005). Das espécies patogênicas, a mais importante é a L. interrogans, com mais de 200 sorovares identificados. Cada um tem os seus hospedeiros preferenciais, mas cada espécie animal pode abrigar um ou mais sorovares. Tais bactérias podem permanecer no solo úmido ou na água por semanas a meses. Entretanto, para manter o seu ciclo vital, necessitam de um hospedeiro animal. 
Os animais são os portadores de leptospiras; o principal é constituído pelos roedores sinantrópicos (ratos domésticos). O Rattus norvegicus é o principal portador do sorovar icterohaemorraghiae (CONFALONIERI, 2005), um dos mais patogênicos para o homem. Reservatórios de menor importância: caninos, suínos, bovinos, equinos, ovinos e caprinos. O modo de transmissão da infecção humana, resulta do contato direto da pele ou mucosa com a urina de animais infectados, principalmente roedores, diluída em coleções hídricas ou águas e lama de enchente (PELISSARI et al., 2011). Raramente pelo contato direto com sangue, tecido, órgão ou urina de outros animais infectados. O período de incubação varia de 1 a 30 dias (em média, de 7 a 14 dias) (SILVA, 2015).

As mudanças climáticas têm aumentado as chuvas torrenciais, furacões e inundações (Nobre et al., 2010), com isso tem aumentado os casos de leptospirose em áreas tropicais do planeta, especialmente em países de menor poder econômico, que têm déficit na vigilância da doença e dificuldade de diagnóstico e notificação não só de casos, como também de óbitos por leptospirose (HARTSKEERL et al., 2011; SCHNEIDER et al., 2013).

Diversos estudos foram realizados no Brasil sobre a incidência da leptospirose (GUIMARÃES et al., 2014; SILVA, 2015; RODRIGUES et al., 2017). No entanto, poucos foram os estudos com destaque ao uso de ferramentas estatísticas descritivas e exploratórias aplicadas a uma série temporal de dados de morbidade e mortalidade. Portanto, o objetivo deste estudo é realizar um diagnóstico da morbidade e mortalidade dos casos de Leptospirose no Nordeste Brasileiro com base em ferramentas estatísticas descritivas e exploratória.

\section{MATERIAL E MÉTODOS}

\section{Área de estudo}

A região do Nordeste Brasileiro (NEB) possui uma área de aproximadamente $1.558 .196 \mathrm{~km}^{2}$ e uma população de 53.081.510 habitantes (IBGE, 2018). O NEB está localizado entre os paralelos de $01^{\circ} 02^{\prime} 30^{\prime \prime}$ e $18^{\circ} 20^{\prime} 07^{\prime \prime} S$ e entre os meridianos de $34^{\circ} 47^{\prime} 30^{\prime \prime}$ e $48^{\circ} 45^{\prime} 24^{\prime \prime}$ W. Limita-se a norte $(N)$ e a leste (E) com o oceano Atlântico, ao sul (S) com os estados de Minas Gerais (MG) e Espírito Santo (ES) e a oeste (W) com os estados do Pará (PA), Tocantins (TO) e Goiás (GO). O NEB é formado por nove estados, são eles: Maranhão (MA), Piauí (PI), Ceará (CE), Rio Grande do Norte (RN), Paraíba (PB), Pernambuco (PE), Alagoas (AL), Sergipe (SE) e Bahia (BA). Os estados podem ser dividos em: Norte do NEB (NNEB) - (MA, PI, CE e RN), Leste do NEB (ENEB) - (PB, PE e AL) e Sul do NEB (SNEB) - (BA).

O NEB tem uma alta variabilidade interanual e intrasazonal da chuva, com a influência térmica dos oceanos Pacífico e Atlântico Tropical, os quais controlam e modulam anos de seca e enchentes na região (ALVES et al., 2017; LYRA et al., 2017). 


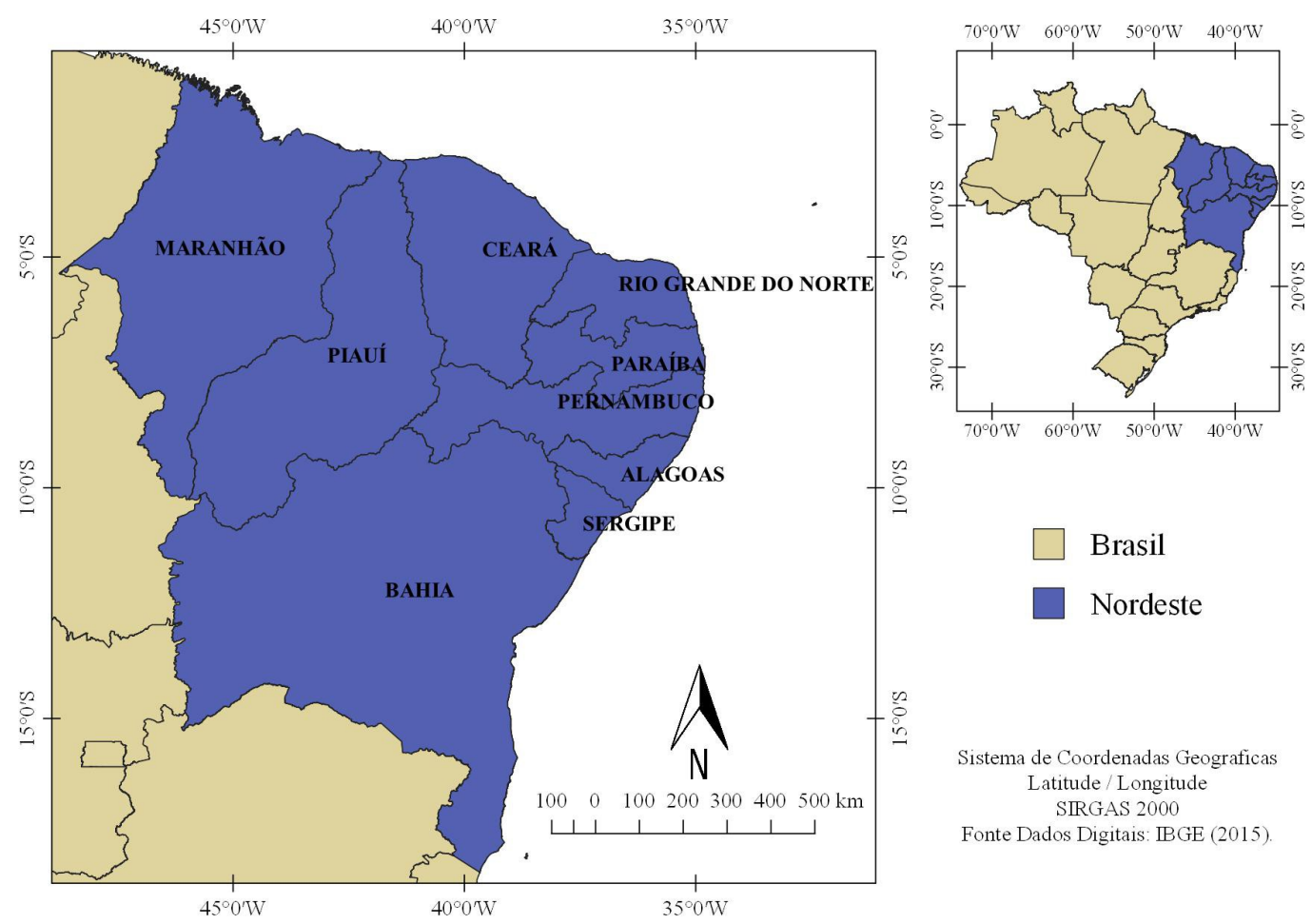

FIGURA 1. Nordeste Brasileiro com seus nove estados, respectivamente.

\section{Série temporal e técnicas estatísticas}

Os dados de morbidade e mortalidade foram obtidos do TABNET a partir do Departamento de Informática do SUS (DATASUS) via endereço eletrônico (http://datasus.saude.gov.br/informacoes-de-saude/tabnet). Na avaliação dos dados de morbidade e mortalidade de dados de leptospirose foram utilizadas ferramentas estatísticas baseadas em análises descritivas (mínimo, máximo, média, desvio padrão, percentagem-\%) e exploratória (boxplot - máximo, mínimo, amplitude interquartílica, primeiro e terceiro quartil, média, mediana (segundo quartil) e valores discrepantes - outliers) da série temporal de dados de morbidade e mortalidade de leptospirose. Vale ressaltar, que todos os procedimentos estatísticos foram realizados no software ORIGIN versão 9.1.

\section{RESULTADOS E DISCUSSÃO}

Baseado na Tabela 1, os anos com maiores casos de morbidade de leptospirose, segundo as médias e os desvios padrão foram 2000 (140,56 $\pm 277,25$ casos), 2004 (89,67 $\pm 114,78$ casos), 2009 (102,78 \pm 98,71 casos), 2010 (79,67 \pm 93,47 casos) e 2011 (102,22 $\pm 117,18$ casos). Vale destacar que, em alguns anos citados anteriormente, ocorreram enchentes no NEB e, portanto, com influência direta no aumento dos casos de morbidade, por exemplo, o ano de 2009 , as chuvas provocaram enchentes principalmente nos estados do $\underline{\mathrm{CE}}$, $\underline{\mathrm{PI}}$, BA, PE e $\underline{\mathrm{MA}}$ (CPTEC/INPE, 2018). A ocorrência de Leptospirose está diretamente relacionada com o período chuvoso, pois, com o aumento do volume de água, a Leptospira é transportada de forma rápida pelo contato com as águas de inundações urbanas (COELHO-ZANOTTI; MASSAD, 2012; GUIMARÃES et al., 2014).

Ao analisar os totais e os percentuais de casos de morbidade apontaram os anos de 2000 (1265 casos, 11,54 \%), 2004 (807 casos, 7,36\%), 2009 (925 casos, 8,44\%), 2010 (717 casos, 6,54\%) e 2011 (920 casos, 8,39\%), como os anos que 
mais se sobressaíram na série temporal. Novamente, os anos citados estão associados aos períodos chuvosos intensos que ocorreram ao longo da série temporal, que por sua vez, determinam as enchentes/inundações, juntamente com aglomeração populacional de baixa renda, em áreas sem infraestrutura e alta infestação de roedores nas Regiões Metropolitanas (RM) do NEB, o que contribuiu para aumento de casos de morbidade (SILVA, 2015). Não se descarta a relação com outro fator, que é o Índice de Desenvolvimento Humano (IDH), que é uma medida resumida do progresso em longo prazo, em três dimensões básicas do desenvolvimento humano: renda, educação e saúde (HAGAN et al., 2016; IBGE, 2018).

TABELA 1. Valores da estatística descritiva (média, máximo, mínimo, percentagem (\%) e total) da morbidade dos casos de leptospirose no NEB no período de 2000-2015.

\begin{tabular}{ccccccc}
\hline Anos & Média & $\begin{array}{c}\text { Desvio } \\
\text { Padrão }\end{array}$ & Máximo & Mínimo & $\begin{array}{c}\text { Percentagem } \\
\mathbf{( \% )}\end{array}$ & Total \\
\hline 2000 & 140,56 & 277,25 & 861 & 2 & 11,54 & 1265 \\
2001 & 72,33 & 101,21 & 324 & 0 & 5,94 & 651 \\
2002 & 70,89 & 96,42 & 307 & 0 & 5,82 & 638 \\
2003 & 57,11 & 66,74 & 201 & 0 & 4,69 & 514 \\
2004 & 89,67 & 114,78 & 371 & 0 & 7,36 & 807 \\
2005 & 82,89 & 114,80 & 335 & 1 & 6,80 & 746 \\
2006 & 75,44 & 74,23 & 224 & 2 & 6,19 & 679 \\
2007 & 63,22 & 67,75 & 205 & 0 & 5,19 & 569 \\
2008 & 71,44 & 59,49 & 193 & 1 & 5,86 & 643 \\
2009 & 102,78 & 98,71 & 303 & 13 & 8,44 & 925 \\
2010 & 79,67 & 93,47 & 271 & 0 & 6,54 & 717 \\
2011 & 102,22 & 117,18 & 383 & 2 & 8,39 & 920 \\
2012 & 45,78 & 39,14 & 119 & 3 & 3,76 & 412 \\
2013 & 58,78 & 70,36 & 187 & 3 & 4,82 & 529 \\
2014 & 62,56 & 68,46 & 220 & 1 & 5,13 & 563 \\
2015 & 42,89 & 44,67 & 125 & 2 & 3,52 & 386 \\
\hline
\end{tabular}

Fonte: DATASUS (2016)

Os anos com maiores casos de mortalidade de leptospirose foram $2000(12,67$ $\pm 23,63$ casos), 2004 (13,33 $\pm 20,48$ casos), 2006 (11,00 $\pm 10,99$ casos), 2009 (9,4 \pm 8,96 caos), 2010 (8,44 $\pm 10,08$ casos) e 2011 (13,11 $\pm 13,57$ casos), conforme as médias e os desvios padrões. Com relação aos totais e os percentuais de casos de mortalidade, os anos de 2000 (114 casos, $11.54 \%$ ), 2004 (120 casos, 7,36\%), 2006 (99 casos, 7,22\%), 2009 (85 casos, 6,20\%), 2010 (76 casos, 5,54\%) e 2011 (118 casos, 8,61\%) - (Tabela 2$)$. 
TABELA 2. Valores da estatística descritiva (média, máximo, mínimo, percentagem (\%) e total) da mortalidade dos casos de leptospirose no NEB no período de 20002015.

\begin{tabular}{ccccccc}
\hline Anos & Média & $\begin{array}{c}\text { Desvio } \\
\text { Padrão }\end{array}$ & Máximo & Mínimo & $\begin{array}{c}\text { Percentagem } \\
(\%)\end{array}$ & Total \\
\hline 2000 & 12,67 & 23,63 & 75 & 1 & 8,32 & 114 \\
2001 & 9,22 & 10,92 & 36 & 0 & 6,05 & 83 \\
2002 & 8,22 & 11,38 & 36 & 0 & 5,40 & 74 \\
2003 & 9,56 & 11,85 & 35 & 0 & 6,27 & 86 \\
2004 & 13,33 & 20,48 & 64 & 0 & 8,75 & 120 \\
2005 & 11,33 & 15,56 & 41 & 0 & 7,44 & 102 \\
2006 & 11,00 & 10,99 & 34 & 0 & 7,22 & 99 \\
2007 & 9,78 & 9,78 & 27 & 0 & 6,42 & 88 \\
2008 & 8,33 & 6,36 & 19 & 0 & 5,47 & 75 \\
2009 & 9,44 & 8,96 & 26 & 0 & 6,20 & 85 \\
2010 & 8,44 & 10,08 & 29 & 0 & 5,54 & 76 \\
2011 & 13,11 & 13,57 & 45 & 0 & 8,61 & 118 \\
2012 & 7,33 & 5,70 & 18 & 0 & 4,81 & 66 \\
2013 & 7,00 & 8,17 & 23 & 0 & 4,60 & 63 \\
2014 & 7,56 & 7,32 & 22 & 0 & 4,96 & 68 \\
2015 & 6,00 & 6,60 & 18 & 0 & 3,94 & 54 \\
\hline
\end{tabular}

Fonte: DATASUS (2016)

Os estados com maiores totais e percentuais de casos de morbidade em Leptospirose no período de estudo foram PE (4506 casos, $41,10 \%)$, seguido da BA (2220 casos, 20,25\%), CE (1248 casos, 11,38\%) e AL (1209 casos, 11,03) - (Figura 2a). Com relação, aos maiores óbitos pela doença nos últimos 15 anos permanecem os estados de PE com 511 casos (10,83\%) e BA 321 casos (15\%), a exceção foi o estado de SE 170 casos (10\%). Os demais estados do NEB a mortalidade foi abaixo de $5 \%$ - (Figura $2 \mathrm{~b}$ ). Os resultados obtidos neste estudo foram similares aos obtidos por Silva (2015) com série temporal de 2000-2013 aplicados ao NEB, as exceções foram a base de dados, tamanho da série temporal e as técnicas estatísticas empregadas, no caso de Silva (2015), apenas o uso da contagem total e média.
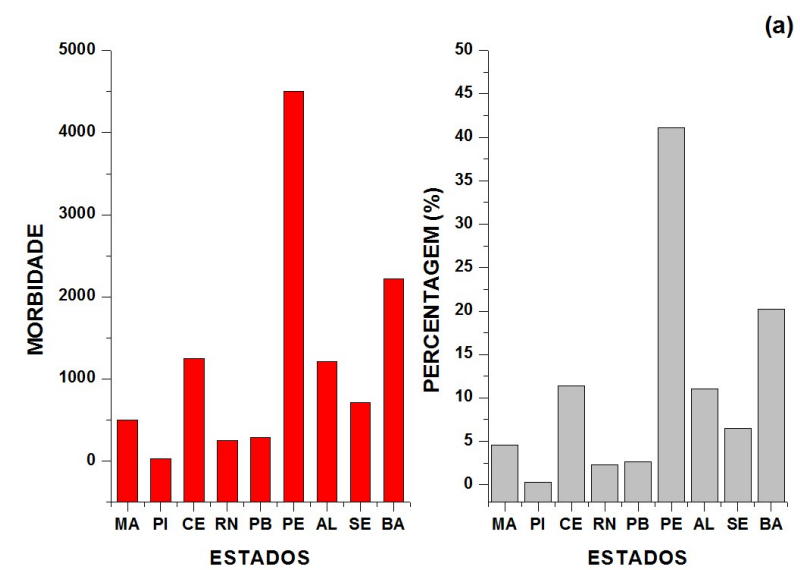

(a)

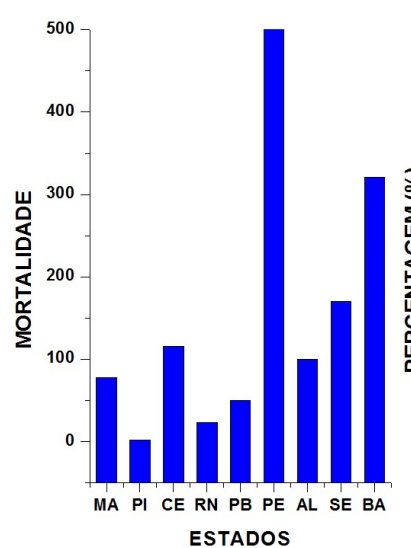

(b)

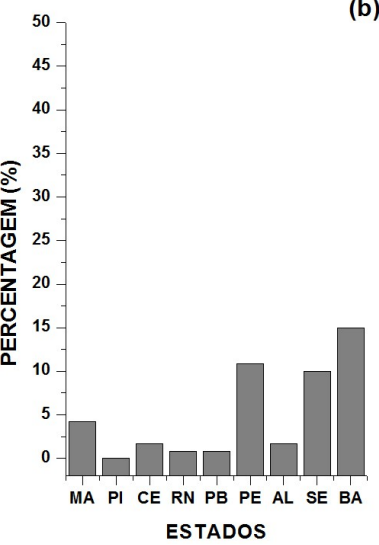

FIGURA 2. Total e percentagem (\%) dos casos de morbidade (a) e mortalidade (b) de casos de leptospirose no NEB no período de 2000-2015. Fonte: DATASUS (2016) 
A distribuição das amostras via boxplot, mostrou uma variabilidade dos casos de morbidade de leptospirose ao longo da série temporal, principalmente, a mediana e amplitude interquartílica. A maioria dos dados foram assimétricos positivos, quando a mediana está próxima ao $1^{\circ}$ quartil, as exceções foram os anos de 2001 , 2008 e 2014 (assimétricos negativos - quando a posição da mediana é próxima ao $3^{\circ}$ quartil) - (Figura 3a). Em toda a série temporal dos casos de morbidade e óbitos de leptospirose, houve outliers (valores discrepantes). Vale destacar que, apenas no ano de 2006, a média e a mediana foram simétricas nos casos de morbidade, situação contrária, ocorreu nos óbitos com anos 2000 e 2006 simétricos. Similarmente aos casos de morbidade, os dados de óbitos de leptospirose foram variáveis a mediana e amplitude interquartílica. Novamente, A maioria dos dados foram assimétricos positivos, seguido das exceções, os anos de 2001, 2008 e 2014 (Figura 3b).
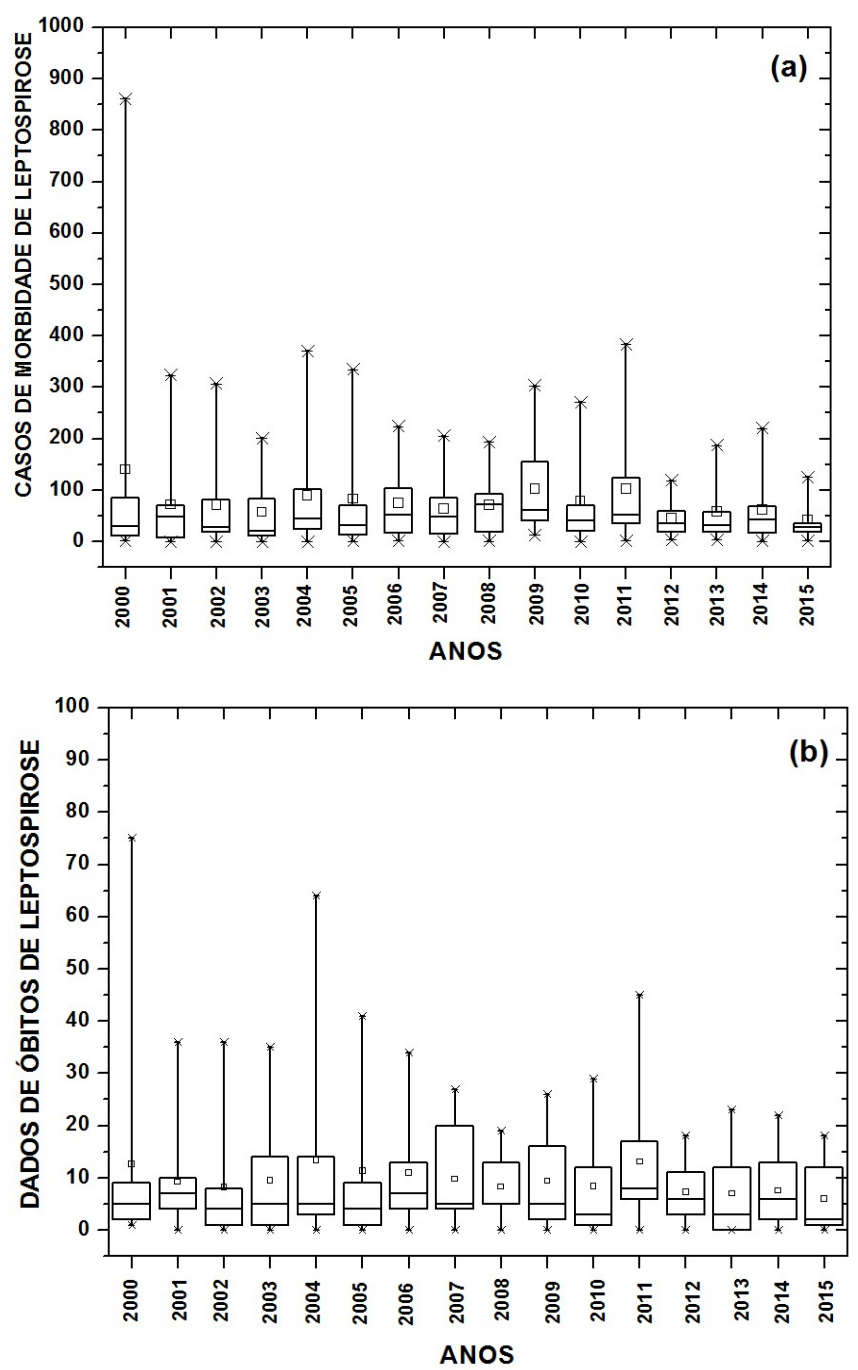

FIGURA 3. Boxplot anual dos casos notificados (a) e de óbitos (b) de leptospirose no NEB no período de 2000-2015. Fonte: DATASUS (2016).

Os estados da $\mathrm{PE}, \mathrm{BA}, \mathrm{CE}$ e $\mathrm{AL}$ se destacaram em relação aos demais com relação aos casos de morbidade de leptospirose. A cidade de Recife (PE) é assolada por ciclos e eventos extremos pluviométricos, tais eventos se repetem nas escalas de 11 anos (2001 e 2012), 7 anos (2004, 2007 e 2014) e 1-2 anos (2001). $\mathrm{Na}$ cidade de Salvador (BA) ocorreram eventos extremos de chuva nos 2000, 2010 e 
2011 e as escalas que potencializam tais extremos de chuvas foram sazonal, interanual curta, 1-2 anos, 7 anos e 22 anos. A cidade de Aracaju (SE) tem comportamento similar ao da cidade de Salvador. A exceção foi Maceió (AL), com escala dominante de 11 anos, isso demonstra uma relação direta entre a chuva local e Dipolo do Atlântico (SILVA et al., 2017), resultado similar encontrado por Lyra et al. (2017). Polzin e Hastenrath (2014) identificaram as escalas preferenciais de 13,2; 9,9 e 5,6 anos na série pluviométrica de Fortaleza (CE), NNEB. Situação contrária ocorreu nos casos de mortalidade, visto que os estados que se sobressaíram foram PE, BA, CE e SE (Figura 4a). Tal variabilidade da chuva nos anos citados anteriormente, juntamente com os problemas das cidades do NEB, algumas RM (Recife, Salvador e Fortaleza) em relação ao grau de urbanização e favelização, contribuíram para aumento substancial da morbidade e mortalidade da leptospirose. Vale destacar que, os estados PE, CE e BA foram assimétricos positivos, a exceção foi AL (assimétrico negativo), no caso das mortalidades ocorreram dados assimétricos negativos - (Figura $4 \mathrm{~b}$ ). Os dados de mortalidade de $\mathrm{AL}$ da série temporal podem ter sido subnotificados. Todos os estados do NEB tiveram outliers na série temporal de casos de morbidade e mortalidade via leptospirose.
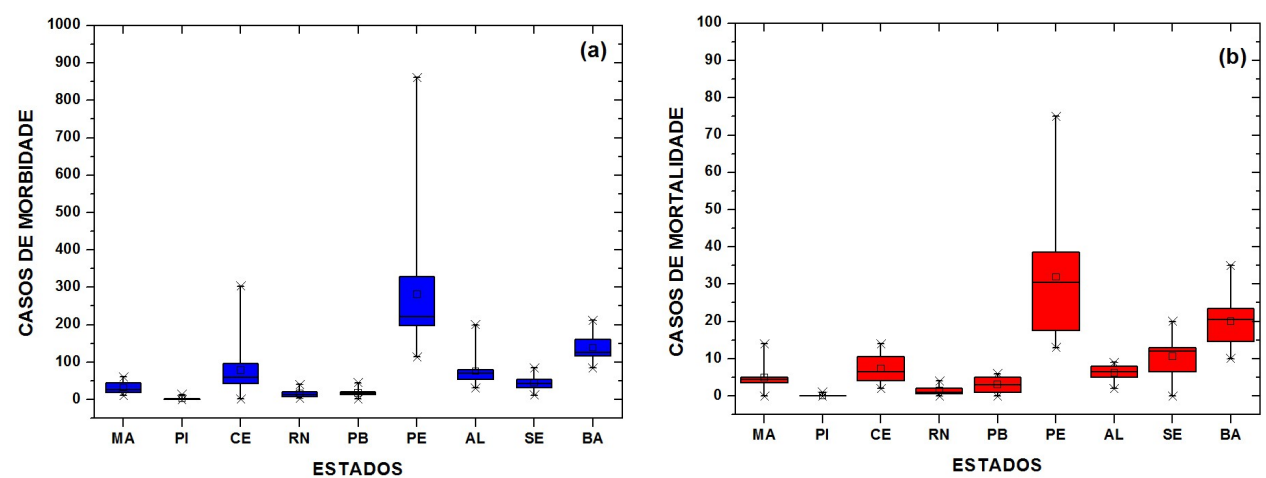

FIGURA 4. Boxplot dos casos de morbidade (a) e de mortalidade (b) de leptospirose por estado no período de 2000-2015. Fonte: DATASUS (2015).

\section{CONCLUSÕES}

A estatística descritiva aplicada à série temporal mostra os anos e os estados com aumento substancial de casos de morbidade e mortalidade da leptospirose no NEB, principalmente no Leste do NEB (ENEB) e engloba os estados da Paraíba, Pernambuco e Alagoas. Os estados de Pernambuco, Bahia, Ceará, Alagoas e Sergipe se sobressaem em relação aos demais na série de 15 anos de dados. A estatística exploratória mostra que a morbidade e mortalidade da leptospirose no NEB são assimétricos positivos, com exceções de alguns anos. Os estados de Pernambuco, Bahia, Ceará, Alagoas e Sergipe se sobressaem, em virtude dos ciclos e eventos extremos pluviométricos que neles ocorrem juntamente com processo de urbanização e o crescimento populacional desordenado no NEB.

\section{AGRADECIMENTOS}

Os autores agradecem ao Centro de Previsão e Estudos Climáticos (CPTEC)/Instituto Nacional de Pesquisas Espaciais (INPE), por ceder gentilmente, os dados climáticos do boletim Climanálise. Ao DATASUS por ceder gentilmente, os dados de morbidade e mortalidade dos casos de leptospirose, no período de estudo via TABNET. 


\section{REFERÊNCIAS}

ALVES, J. M. B., SILVA, E. M., SOMBRA, S. S., BARBOSA, A. C. B., SANTOS, A. C. S.et al. Eventos Extremos Diários de Chuva no Nordeste do Brasil e Características Atmosféricas. Revista Brasileira de Meteorologia, v. 32, n. 2, p. 227-233, 2017. Disponível em: https://dx.doi.org/10.1590/0102-77863220012. doi: $10.1590 / 0102-77863220012$

CLEMENTE, S. S., OLIVEIRA JÚNIOR, J. F. A importância do ser humano de se reconhecer no Lugar natureza, segundo a perspectiva da educação ambiental. Educação Ambiental e Humanidade: A harmonia de um mosaico. $1^{\text {a }}$ ed.Rio de Janeiro: Agenda Dinâmica, v. 1, p. 107-118, 2015.

COELHO-ZANOTTI, M. S. S.; MASSAD, E. The impact of climate on Leptospirosis in São Paulo, Brazil. International Journal of Biometeorology, v. 56, n. 2, p.233-41, 2012. Disponível em: https://doi.org/10.1007/s00484-011-0419-4. doi: 10.1007/s00484-011-0419-4

CONFALONIERI, U. E. C. Saúde na Amazônia: um modelo conceitual para a análise de paisagens e doenças. Estudos Avançados, v.19, n. 53, p.221-236, 2005. Disponível em: https://dx.doi.org/10.1590/S0103-40142005000100014. doi: 10.1590/S0103-40142005000100014

CPTEC/INPE. Centro de Previsão de Tempo e Estudos Climáticos/ Instituto Nacional de Pesquisas Espaciais - Climanalise. Disponível no site em: http://climanalise.cptec.inpe.br/ rclimanl/boletim/. Acesso em 10 de março de 2018.

DATASUS - DEPARTAMENTO DE INFORMÁTICA DO SUS. Informações de Saúde, Epidemiológicas e Morbidade: banco de dados. Disponível no site em: http://datasus.saude.gov.br/informacoes-de-saude/tabnet. Acesso em 21 de janeiro de 2016.

DUTRA, F. R. L. S., VALADÃO, R. C., CONFALONIERI, U. E., MULLER, G. V., QUADRO, M. F. L. A influência da variabilidade da precipitação no padrão de distribuição dos casos de leptospirose em Minas Gerais, no período de 1998 20121. Hygeia, v. 11, n. 20, p. 106-126, 2015.

GONÇALVES, N. V., ARAÚJO, E. N., SOUSA JÚNIOR, A. S., PEREIRA, W. M. M., MIRANDA, C. S. C., et al. Distribuição espaço-temporal da leptospirose e fatores de risco em Belém, Pará, Brasil. Ciência \& Saúde Coletiva, v.21, n.12, p. 3947-3955, 2016. Disponível em: https://doi.org/10.1590/1413-812320152112.07022016. doi: 10.1590/1413-812320152112.07022016

GOULART, A. C. O.; DELGADO, R. C.;OLIVEIRA JÚNIOR, J. F.; GOIS, G.; OLIVEIRA, E. S. Relação espectro-temporal entre índices de vegetação e a chuva na cidade do Rio de Janeiro. Revista de Ciências Agrárias (Belém), v. 58, n. 3, p. 277-283, 2015. Disponível em: http://dx.doi.org/10.4322/rca.1990. doi: $10.4322 /$ rca. 1990

GUIMARÃES, R. M.; CRUZ, O. G.; PARREIRA, V. G.; MAZOTO, M. L.; VIEIRA, J. D., et al. Análise temporal da relação entre leptospirose e ocorrência de inundações 
por chuvas no município do Rio de Janeiro, Brasil, 2007-2012. Ciência \& Saúde Coletiva, v. 19, n. 9, p. 3683-3692, 2014. Disponível em: https://dx.doi.org/10.1590/1413-81232014199.06432014. doi: 10.1590/141381232014199.06432014 
HAGAN, J.E.; MORAGA, P.; COSTA, F.; CAPIAN, N.; RIBEIRO, G.S., et al. Spatiotemporal Determinants of Urban Leptospirosis Transmission: Four-Year Prospective Cohort Study of Slum Residents in Brazil. PLoS Neglected Tropical Diseases, v.10, n. 1, p. 1-16, 2016. Disponível em: https://doi.org/10.1371/journal.pntd.0004275. doi: 10.1371/journal.pntd.0004275

HARTSKEERL, R. A.; COLLARES-PEREIRA, M.; ELLIS, W.A. Emergence, control and re-emerging leptospirosis: dynamics of infection in the changing world. Clinical Microbiology and Infection, v. 17, n.4, p. 494-501, 2011. Disponível em: https://doi.org/10.1111/j.1469-0691.2011.03474.x. $\quad$ doi: $10.1111 /$ j.14690691.2011.03474.x

IBGE. Instituto Brasileiro de Geografia e Estatística. Disponível em http://www.ibge.gov.br/. Acesso em 10 de março de 2018.

LYRA, G. B.; OLIVEIRA-JÚNIOR, J. F.; GOIS, G.; CUNHA-ZERI, G.; ZERI, M. Rainfall variability over Alagoas under the influences of SST anomalies. Meteorology and Atmospheric Physics (Print), v. 129, n. 2, p. 157-171, 2017. Disponível em: https://doi.org/10.1007/s00703-016-0461-1. doi: 10.1007/s00703016-0461-1

NOBRE, A. C., YOUNG, A. F., SALDIVA, P., MARENGO, J. A., NOBRE, A. D., et al. Vulnerabilidades das megacidades brasileiras às mudanças climáticas: Região Metropolitana de São Paulo. Sumário Executivo, INPE, IPT, UNICAMP, USP, UNESP- Rio Claro. Junho. 2010. Disponível em: http://mudancasclimaticas.cptec.inpe.br/ rmclima/pdfs/publicacoes/2010/SumarioEx ecutivo_me gacidades.pdf. Acesso em: 20 de março de 2018.

PELISSARI, D. M; ELKHOURY, A. N. S. M.; ARSKY, M. L. N. S, NUNES, M. L. Revisão sistemática dos fatores associados à leptospirose no Brasil, 2000-2009. Epidemiologia e Serviços de Saúde, v. 20, n. 4, p. 565-574, 2011. Disponível em: http://dx.doi.org/10.5123/S1679-49742011000400016. doi: 10.5123/S167949742011000400016

POLZIN, D.; HASTENRATH, S. Climate of Brazil's Nordeste and Tropical Atlantic Sector: Preferred Time scales of variability. Revista Brasileira de Meteorologia, v. 29, n. 2, p. 153-160, 2014. Disponível em: http://dx.doi.org/10.1590/S010277862014000200001. doi: http://dx.doi.org/10.1590/S0102-77862014000200001

RODRIGUES, C. M. Sobre leptospirose e informação: ampliando os conceitos de negligência em saúde. ABCS Health Sciences, v. 42, n. 1, p. 45-49, 2017. Disponível em: http://dx.doi.org/10.7322/abcshs.v42i1.949. doi: 10.7322/abcshs.v42i1.949

SCHNEIDER, M. C., JANCLOES, M.; BUSS D.F.; ALDIGHIERI, S.; BERTHERAT, E., et al. Leptospirosis: a silent epidemic disease. International Journal Environmental Research and Public Health, v. 10, n. 12, p. 7229-34, 2013. Disponível em: http://dx.doi.org/10.3390/ijerph10127229. doi: 10.3390/ijerph10127229 
SILVA, D. F. Aplicação de Análises de Ondaletas para Detecção de Ciclos e Extremos Pluviométricos no Leste do Nordeste do Brasil. Revista Brasileira de Meteorologia, v. 32, n. 2, p. 187-198, 2017. Disponível em: https://dx.doi.org/10.1590/0102-77863220002. doi: 10.1590/0102-77863220002

SILVA, G. A. Enfoque Sobre a Leptospirose na Região Nordeste do Brasil Entre os Anos de 2000 A 2013. Acta Biomedica Brasiliensia, v.6, n.1, p. 101-108, 2015.

SOUSA M. I. F., BARBOSA J. J., COSTA, C. T. F. Uma reflexão sobre mudanças climáticas, saúde e meio ambiente no semiárido nordestino. Revista Saúde \& Meio Ambiente, v. 4, n. 2, p. 61-77, 2015. Disponível em: http://dx.doi.org/10.24302/sma.v4i2.802. doi: 10.24302/sma.v4i2.802 